CapiPay: A System for Providing Capitation Payments to Research Centers Involved in a Multicenter Clinical Research Network

Jeanette Auman and Callie Riggs

January 2015 


\section{About the Authors}

Jeanette Auman, BS, a research programmer/analyst, has been a computer programmer at RTI International since 1998. She specializes in developing electronic data capture systems, managing study data, and leading the RTI technical team on a multi-center research project.

Callie Riggs, MBA, is a research health analyst in RTI's Center for Clinical Research Network Coordination. She has managed the contractual, administrative, and fiscal affairs of many multisite research projects. She also manages capitation payments for several multisite, multiple-protocol projects.
This publication is part of the RTI Research Report series. Occasional Papers are scholarly essays on policy, methods, or other topics relevant to $\mathrm{RTI}$ areas of research or technical focus.

RTI International

3040 East Cornwallis Road PO Box 12194

Research Triangle Park, NC 27709-2194 USA

Tel: $\quad+1.919 .541 .6000$

Fax: $\quad+1.919 .541 .5985$

E-mail: rtipress@rti.org

Website: www.rti.org
RTI Press publication OP-0021-1501

This PDF document was made available from www.rti.org as a public service of RTI International. More information about RTI Press can be found at http://www.rti.org/rtipress.

RTI International is an independent, nonprofit research organization dedicated to improving the human condition by turning knowledge into practice. The RTI Press mission is to disseminate information about RTI research, analytic tools, and technical expertise to a national and international audience. RTI Press publications are peer-reviewed by at least two independent substantive experts and one or more Press editors.

\section{Suggested Citation}

Auman, J., and Riggs, C. (2015). CapiPay: A System for Providing Capitation Payments to Research Centers Involved in a Multicenter Clinical Research Network (RTI Press publication OP-0021-1501). Research Triangle Park, NC: RTI Press.

(C)2015 Research Triangle Institute. RTI International is a trade name of Research Triangle Institute.

All rights reserved. This report is protected by copyright. Credit must be provided to the author and source of the document when the content is quoted. Neither the document nor partial or entire reproductions may be sold without prior written permission from the publisher.

http://doi.org/10.3768/rtipress.2015.op.0021.1501 


\title{
CapiPay: A System for Providing Capitation Payments to Research Centers Involved in a Multicenter Clinical Research Network
}

\author{
Jeanette Auman and Callie Riggs
}

\section{Abstract}

In a research setting, capitation is a payment made to a clinical center for completing a study-related task. Capitation is the main avenue for the National Institutes of Health $(\mathrm{NIH})$ to reimburse research costs to research centers on a per-patient-enrolled basis. This paper describes the design, building, and implementation of the capitation system, CapiPay, for the Eunice Kennedy Shriver National Institute of Child Health and Human Development (NICHD) Neonatal Research Network (NRN), a network of clinical research centers conducting multicenter clinical and observational studies.

This report details the process of RTI, as the NRN Data Coordinating Center (DCC), assuming responsibility for capitation payments from the funding agency, NICHD, and designing a system that produces accurate and timely payment to the clinical research centers. We also describe the accounting decisions and considerations made in developing this system. This process required working with NIH, RTI project staff, various research and administrative groups within RTI, and the clinical research centers to implement a new payment method. Our goal was to make the transition appear seamless to the research centers and to $\mathrm{NIH}$. The final product is a system that has benefitted $\mathrm{NIH}$ and the research centers and that can be customized for use on other DCCs by RTI. 


\section{Introduction}

Research clinical trials are considered the gold standard of clinical research and are integral to evidence-based practice of medicine and comparative effectiveness studies.

In the randomized clinical trial setting, capitation payments are made to centers for their involvement in clinical trials. These payments are the principal means by which academic medical centers typically recoup their research study costs on a per-patient basis. This reimbursement mechanism also may incentivize patient enrollment and prompt data entry, thus facilitating speedier recruitment and completion of clinical trials. However, trials are becoming increasingly complicated to run, recruit into, finish, and publish on time (National Academy of Sciences, 2010). Given this backdrop and the erosion of federal research funding (Harris \& Benincasa, 2014), clinical centers must be properly and efficiently reimbursed for their study costs and incentivized for ramping up recruitment. At the same time, government cost-accounting standards require well-documented financial transactions. Thus, paying participating research centers for enrolling patients must be efficient and transparent.

Very little has been written about the method or system of providing capitation payment to research centers. Recently, data coordinating centers (DCCs) have been tasked with managing the capitation funds provided to research centers for enrolling study participants and other study-related items. This paper provides information about challenges of capitation fund implementation and management. In particular, we provide insight into the system we designed for the Neonatal Research Network (NRN). This insight is intended to inform DCCs that are beginning to implement a capitation payment process as well as DCCs that have a system in place but are looking for process improvements.

We discuss transitioning capitation payments from the Eunice Kennedy Shriver National Institute of Child Health and Human Development (NICHD) to the project data coordinating center, including accounting and contractual specifications. We describe how capitation payments are managed, maintained, and provided to multiple research centers participating in multiple clinical trials via a custombuilt capitation software system. Additionally, we discuss the benefits of this transition to this project's DCC, the funding agency, and the research centers.

\section{Background}

RTI has acted as the DCC on numerous multicenter health-related research projects for more than 30 years. These projects often involve a network of research centers working together on various studies to help improve the human condition. As the DCC for these types of projects, RTI works with sponsors and clinical investigators to create randomized clinical trials aimed toward a common goal. The DCC collaborates with investigators to create data collection instruments and study materials, provides data management and data analysis, coordinates research meetings, develops manuscripts, provides Institutional Review Board management and expertise, tracks biospecimen samples, and provides specialized reports to the funding agencies. Typically, the DCC maintains a central repository of all centers' study data.

\section{Neonatal Research Network}

RTI provides statistical and data coordinating services to the NICHD NRN, a cooperative group of 18 actively enrolling and 4 collaborating US hospitals. These hospitals enroll premature babies into clinical trials and observational studies that test the efficacy and safety of various treatments, with several protocols active at any given time. The NRN has been functioning for more than 20 years, with RTI as the DCC since 1998.

In 2009, NICHD notified RTI that it would transfer management of capitation funds to RTI. This was the first project for which RTI was responsible for tracking the enrollment for capitation and distributing the capitation funds to research centers. At the time of publication, the NRN manages six randomized clinical trials, two observational studies, multiple secondary studies, and four follow-up studies. Each study receives at least one type of capitation payment per enrollment. 


\section{What Is Capitation?}

In a research setting, capitation is a payment the funder makes to a research center for completing a task. The payment often is related to enrolling a patient into a research study. However, payments may also be related to individual tasks within a research study, such as obtaining an MRI or completing an interview. Additionally, a capitation payment can be for other research-related costs, such as attending a training session, pharmacy set-up for a new study, or the purchase of equipment or materials necessary for conducting the study. The capitation payment amounts are fixed by study or task. The umbrella of what may be considered capitation can be fairly large and ambiguous.

Historically on the NRN, each research center enrolled study participants and entered study responses into an electronic data capture tool (EDC). RTI maintained a central repository of all study data. When NICHD managed capitation, funding was distributed to the research centers annually. NICHD provided funds at the beginning of the project year based on historic rates of enrollment and, as they enrolled patients, the research centers deducted funds from the amount. At the end of the year, NICHD reconciled the amounts that it provided to each center. RTI produced specialized enrollment reports from the central data repository for each funding period so that NICHD could determine study enrollment and the correct amount for capitation payments. When developing our capitation system, CapiPay, we sought to streamline this process and make it less prone to human error.

\section{Transitioning Capitation Responsibility}

Planning and implementing the capitation payment system for the NRN was a year-long process. We were given a "go live" date, and we had 1 year to gather information, collaborate with all interested parties, and then plan, develop, test, and implement a functional system. Implementing a new payment system required significant collaboration across RTI and with NICHD. We worked closely with RTI Corporate Accounting and Accounts Payable, Office of Contracts, DCC staff, NRN research staff, and NICHD to develop a system that met everyone's needs.

Before beginning to make payments to research centers, we made several internal decisions about how to classify the research centers as vendors and how to account for the capitation funds that RTI would receive. After in-depth consideration, the funding was considered pass-through funding rather than revenue. To simplify the contractual process with the research centers, memoranda of understanding (MOUs) were developed to outline the roles and responsibilities for RTI and the research centers. The MOUs were signed by the responsible RTI contracts office member and a corresponding grant manager at the clinical centers and are renewed annually as each year of funding is received. We can make modifications and amendments for several reasons, such as a change in period of performance or cofunding from other government agencies.

\section{Payment Process}

Making a capitation payment is different from paying a subcontractor's invoice. The primary difference is that subcontractors generate their own invoices and submit them to us for payment. With our payment process, we are responsible for generating the statements and making the payments. The only action required by the research centers so they can receive payment is for them to enroll patients and enter the enrollment data into the EDC. The billing statement is generated from our CapiPay system. After a quality assurance review, the statements are approved by the project principal investigator, then submitted to RTI's Accounts Payable department for payment. The payments are processed quickly to ensure timely payment to the centers.

\section{Computer System Specifications}

After determining the internal processes of how we would pay capitation, we next designed and built the capitation computer system. We determined that the capitation system must produce payments for items previously mentioned: (1) patient enrollment into various randomized clinical trials, (2) collection of study-specific measurements such as MRIs 
and ultrasounds, (3) various other study-related items such as follow-up visits and participation in secondary studies, and (4) other direct costs, for example, study training, equipment purchase, and lab start-up fees. The criteria used to determine whether a payment is made are defined by the study principals, such as a steering committee, the principal investigator, or the RTI project director. We refer to the criterion for payment as the "capitation trigger."

We made some initial assumptions for the system, including (1) the system will access some electronic study data, (2) the system will be activated to produce invoices at specified time intervals, (3) payments will be made only once for each occurrence of a capitation trigger being met, (4) historical data will be stored in case of audit, and (5) payments will be added as new clinical trials start throughout the project.

\section{External Operational Factors}

We had to answer questions about external factors affecting the design of CapiPay. How often do we receive study data? Would any computer-assigned fields be helpful to use with the capitation payments? How thoroughly and completely are the data entered into the data capture tool? Should any scheduling or other issues be factored into our system design?

For the NRN project, each research center sends study data to RTI via secure data transmission. The NRN EDC assigns creation and completion flags to each data record, which we use to trigger payments. The creation date is when the data are initially created in the EDC. This system date is helpful in determining which capitation period the data record is paid. We assign a completion flag to a data record after programmed quality checks are successfully applied to the record. This flag indicates that the data are complete and satisfy all programmed quality checks. We decided to make payments 2 months after the data center enters the case into the EDC to allow time for resolution of data edits, deletion of duplicate records, or entry of cases that were mislabeled as eligible. For example, we pay centers in October for cases entered in August, which gives them 1 month, September, to correct errors.

We built CapiPay using SAS software. The project data files are regularly converted from the EDC to SAS data sets for use by study analysts. On the NRN, a preexisting monthly report programmed in SAS software provides enrollment numbers to the different research centers and the funding agency for all current studies. Therefore, we programmed the capitation system in SAS for optimized efficiency so we could use some of the same programming code to ensure system compatibility.

The NRN has a project website that is divided into a public and a private portal. The private portal is accessible by individual user name and password. Each user is assigned access to specific areas within the private portal based on his or her user role. We decided that the capitation invoices, reports, and other relevant information would be housed on the private portal of the NRN website and made accessible to a group of users who have permission to access the finance portion of the website.

\section{Functional Requirements}

The capitation system houses other necessary capitation information: other direct cost payments, research center financial information, and descriptions of capitation triggers. The system uses the study data and other capitation data to produce reports and invoices that detail the capitation payments made to the research centers.

\section{Manage and House All Information Used by CapiPay}

Not only does the capitation system use study data, it also must use research centers' financial information and other direct cost payments. The system uses specific research center information, such as center name, contact information for financial staff, center indirect rates, grant numbers, and addresses, to produce invoices and provide payment for the center's indirect costs. Because networks tend to consist of studies that continue over a number of grant funding cycles, the research centers involved in the NRN may change. Information about a particular center, such as its contact information or indirect rates, may change as well. The capitation system houses this information and allows system users to edit it.

The capitation system must pay other direct costs that cannot be determined from the study data. These costs can include items like study training, study 
equipment, and pharmacy fees. The capitation system stores these payments and adds them to the center's invoices.

To store this additional information, we use a password-protected relational database that is accessed only by the capitation system programmer and financial administrator for the project. We limit access to the database to prevent inadvertent altering of the payment data.

\section{Ensure That All Payments Are Captured}

Because delays can occur between study data collection, entry, and transmission to RTI, CapiPay identifies all eligible records for payment, including those with dates before the specified capitation time period. These eligible payments are compared with past payments, and only new ones are added to research center invoices. This method ensures that no eligible payments are missed no matter how late they are received. The method also allows the NRN's Steering Committee to easily add new payments after a study begins enrolling.

\section{Data Changes Resulting in Subtraction}

Research staff can change their study data after initial submission to RTI. This presents a potential problem, because data that triggered a payment could be changed to be no longer eligible. The capitation system addresses these potential data changes and subtracts the past payment from the invoice. The system also provides a report for RTI staff to verify these subtractions before they are sent to the research center. Any indirect costs associated with a payment are also deducted with the subtraction.

\section{Output Summaries and Historical Payment Data}

To ensure payment transparency, the output of the capitation system is auditable and clearly defined. The research center's billing statement lists each capitation payment with an item count and total payment columns for the month, the project year, and cumulative for the period of project capitation(as shown in Figures 1 and 2). The system also outputs summary reports listing all payments made for the time period and an item count for each capitation

\section{Figure 1. Example page 1 of billing statement}

09:54 Wednesday, January 14, $2015 \quad 1$

\section{NICHD Neonatal Research Network \\ Monthly Site Capitation Statement}

Hospital Name

Jane Doe

123 Walt Disney Lane

Orlando, FL 99999
Date Prepared: 08/09/2013

Capitation Period: 06/01/2013 - 06/30/2013

Statement Number: U99HD9999-1308

Vendor ID: T9999

\begin{tabular}{|c|c|c|c|c|c|c|c|}
\hline \multicolumn{2}{|c|}{ Capitation Payment Items } & \multicolumn{2}{|c|}{$\begin{array}{c}\text { Current } \\
\text { Capitation } \\
\text { Period }\end{array}$} & \multicolumn{2}{|c|}{$\begin{array}{c}\text { Project YTD } \\
(04 / 01 / 2013-03 / 31 / 2014)\end{array}$} & \multicolumn{2}{|c|}{$\begin{array}{l}\text { Cumulative } \\
\text { To Date }\end{array}$} \\
\hline \multirow[t]{2}{*}{ Study A } & $\$ 105.00$ & . & . & . & & 99 & $\$ 10,395.00$ \\
\hline & $\$ 120.00$ & 6 & $\$ 720.00$ & 10 & $\$ 1,200.00$ & 12 & $\$ 1,440.00$ \\
\hline Study C & $\$ 1,900.00$ & 1 & $\$ 1,900.00$ & 1 & $\$ 1,900.00$ & 1 & $\$ 1,900.00$ \\
\hline Study A Supplies & $\$ 50.00$ & . & . & . & & 1 & $\$ 50.00$ \\
\hline Study C Training costs & $\$ 2,000.00$ & . & . & . & & 1 & $\$ 2,000.00$ \\
\hline \multicolumn{2}{|c|}{ Total Amount Due for Payment: } & \multicolumn{2}{|c|}{$\$ 7,488.90$} & & & & \\
\hline
\end{tabular}


Figure 2. Example page 2 of billing statement

09:54 Wednesday, January 14, $2015 \quad 2$

NICHD Neonatal Research Network

Monthly Site Capitation Statement

Hospital Name

Jane Doe

123 Walt Disney Lane

Orlando, FL 99999
Date Prepared: 08/09/2013

Capitation Period: 06/01/2013 - 06/30/2013

Statement Number: U99HD9999-1308

Vendor ID: T9999

\begin{tabular}{|c|r|}
\hline Project Charge Code & Amount \\
\hline $\mathbf{0 9 9 9 9 9 9 . 9 9 9 . 0 0 1}$ & $\mathbf{\$ 7 , 4 8 8 . 9 0}$ \\
\hline & $\$ 7,488.90$ \\
\hline
\end{tabular}

trigger. The capitation system maintains a historical data set of all payments made and any relevant information about each payment for the span of the project. We use this data set to produce various summary reports so that all money paid is accounted for during the life of the project.

\section{Easy Addition of New Capitation Triggers}

Multisite projects continuously implement new studies. In the NRN, often multiple clinical trials enroll patients consecutively, and each trial has its own criteria for capitation. Therefore, we built the capitation system using a modular approach, which allows us to easily add new capitation payments without affecting existing payments. We programmed study payments or triggers into a micro-program, which produces a data set containing records eligible for payment. We add these records to the total list of payments. This programming style allows us to easily insert individual capitation trigger programs into the larger capitation system.

\section{Transparency}

Transparency was very important to us as we began the capitation payment process, and we created several documents to keep the process as clear as possible for the research centers. Our Detailed Capitation Information (Capi Info) document, used to answer questions about capitation, is a living reference document and is therefore revised on an ongoing basis. The Capi Info document clearly states the capitation rate for each study, what that rate is based on (such as nurse coordinator labor hours, labor plus lab tests), which form needs to be completed, and how specific study form questions must be answered for a center to receive the payment. We update the Capi Info document as studies are added or completed, when a capitation rate is changed, or when additional information would be useful for the research centers. The document is posted on the private portal of the project website.

We also provide the research centers with backup documentation for each payment we make. We post a copy of the centers' billing statements (Figures 1 and 2) on a research center-specific page of the project's website. Research center staff can only access their own center's billing statements. The billing statements are accompanied by a patient ID list, which allows the centers to track their payments by the patient and the study. If research staff have any questions, they can review the data entered into the EDC with the specified record identifier.

The capitation system can also generate an additional center report: a list of record identifiers that were subtracted from a center's invoice. This report is only generated if a subtraction occurs during the current capitation period.

Finally, we share the financial contact information for each research center, including RTI's contact 
information. Although RTI works closely with the research centers and RTI Accounting to set up the payment process for each center, the research centers themselves are not always accustomed to receiving this type of payment. Therefore, the center investigators may not be in regular contact with their accounting departments or grants offices, which can lead to difficulty ensuring that the payments get to the correct accounts. We post the financial contact information for each center so that they can easily identify who to contact when they have questions.

We generate a quarterly capitation summary report to help RTI and the research staff better plan for future studies and plan how to further invest capitation funds. This report lists all payments within the current project year and forecasts the amount to be spent for the remainder of the project year based on previous enrollment. For those studies that are not yet currently enrolling, RTI staff review and estimate potential enrollment.

\section{Lessons Learned and System Enhancements}

The billing statement number has become a key piece of information for the research centers. During the first months of capitation payments, the centers' accounts receivable and finance staff asked numerous questions, such as what the funds were for and how to apply them to the correct account. We modified the statement number to reflect each center's grant number, followed by the date as YYMM. The statement number is included with the electronic funds transfer for centers that receive electronic payments and is printed on hardcopy checks as well. Including the grant number in the billing statement number ensures that the recipient's finance office can locate and apply the funds to the correct account because each research study has its own grant or contract number.

We have already instituted a change to CapiPay for the NRN that allows us to charge to multiple accounting codes within one invoice. Previously, it was assumed there would be only one accounting code for the entire project. However, as funding changes were made over time, we were required to charge specific accounting codes for different studies within the NRN project. The invoice now shows the various dollar amounts for each study accounting code used for payment.

Because similar data coordinating projects have begun to implement the CapiPay system, project-specific functionality has been added. One such feature was the addition of a payment "type" used to differentiate between a lump sum payment and a unit rate, such as an hourly rate. With this new feature, RTI can pay a rate multiplied by the amount of units reported in the data.

We are still exploring certain limitations to CapiPay. One is the current size of the historical payment data set, which will continue to grow over time. The CapiPay system has been used to distribute more than $\$ 11$ million to NRN research centers in its first 4 years. During this time, the historical data set has grown considerably, housing every past study payment and trigger, as well as financial information. For example, there are approximately 8,500 records of payments for just one of the NRN's main studies. This study will continue to enroll throughout the life of the project. Because of this, we are discussing ways to break the data set down to make it more manageable and dynamic. One initial thought is to divide payments by the project funding cycle so that the data set is cumulative for only a set number of years.

One other limitation involves subtracted payments. If a research center does not receive payment for a month and changes data to reflect a subtraction, its invoice might have a negative balance. Currently, CapiPay is programmed under the assumption that any negative payments will be recouped within the current invoice. Therefore, we must modify it in a future version so that these negative payments are not lost but, rather, pushed to the next invoice with a positive balance. When the center enters future data, we can recoup that subtracted dollar amount. If after a certain time it does not recoup that amount, we can request reimbursement from the center.

Finally, we plan to survey the research center staff to obtain feedback for future system enhancements. We think one request will be to post all yearly invoices to the project website so centers may review them as needed. 


\section{Discussion}

Before RTI managed capitation for the NRN, centers were responsible for paying themselves as they enrolled study participants. Therefore, research staff were required to make assumptions about data in the central repository used for analysis. There were no formal definitions of payment triggers or which date value to use to determine in what payment period a payment should be applied. The sponsor would reconcile capitation payments annually based on enrollment reports from RTI, and these reports often did not correspond to the research centers' enrollment records. Assumptions about the completeness of the data, data values that constituted a payment, when the data were entered, or which capitation year a patient should be included in if enrolled on the cusp of that year often explained why the research center and sponsor reports would disagree.

With our capitation process, research centers receive monthly statements clearly outlining which studyrelated payment they are receiving. Centers can reconcile their payments using the billing statement and study ID report rather than assumptions. This significantly benefits the research centers because it reduces the amount of time required to review and reconcile capitation payments. The research centers can focus on enrolling patients and participating in research. If the centers are subjected to an internal or external audit, they will have clear documentation to support the payments they received.

The previous capitation payment method also required research centers to manage their lump payments. This can be difficult because it necessitates careful budgeting. It also relied completely on enrollment forecasts for the research center's annual capitation payment. If a center did not enroll as originally expected, it would either be short of funds by the end of the year or would have underspent its funding and be required to carry remaining funds forward or return them to the sponsor. Monthly CapiPay payments provide a steady cash flow to the centers, which eases the strain of budgeting. The sponsor is also relieved not only of the burden of accounting for the funds on a regular basis but also of audits for managing these funds.

Tying the payments directly to the study data may also motivate research staff to perform timely data entry. Centers are only paid for enrollment if the data are entered into the EDC. Centers are more likely to enter their data in a timely manner so that they are paid promptly, and RTI benefits by having access to the data without delay.

The payment process is transparent from start to finish. The Capi Info document clearly explains what the centers need to do to receive payment and the amount they will be paid for each study. As each monthly statement is created, it is posted online for the centers to review, along with the corresponding patient IDs. We e-mail the centers after the payments are made so that they will know when they will receive an EFT or when they can expect to receive a check. Transparency was a primary goal from the beginning, and the reports and communications we provide have helped to achieve that goal.

The end result of taking on the capitation payment responsibility is a system that reduces administrative work load for the government sponsor and the research centers and provides a high degree of transparency for all monetary transfers.

\section{References}

Harris, R., \& Benincasa, R. (2014). US science suffering from booms and busts in funding [Article online]. Retrieved January 5, 2015, from http://www. npr.org/blogs/health/2014/09/09/340716091/us-science-suffering-from-booms-and-busts-infunding
National Academy of Sciences. (2010). Transforming clinical research in the United States: Challenges and opportunities: Workshop summary. Washington, DC: National Academies Press. Retrieved January 8, 2015, from http://www.ncbi. nlm.nih.gov/books/NBK50888/ 


\section{Acknowledgments}

The authors wish to acknowledge the Eunice Kennedy Shriver National Institute of Child Health and Human Development for funding the Neonatal Research Network. In addition, the authors acknowledge Abhik Das, $\mathrm{PhD}$, and Philip Cooley, MS, of RTI International for their review and valuable input. 
RTI International is an independent, nonprofit research organization dedicated to improving the human condition by turning knowledge into practice. RTI offers innovative research and technical solutions to governments and businesses worldwide in the areas of health and pharmaceuticals, education and training, surveys and statistics, advanced technology, international development, economic and social policy, energy and the environment, and laboratory and chemistry services.

The RTI Press complements traditional publication outlets by providing another way for RTI researchers to disseminate the knowledge they generate. This PDF document is offered as a public service of RTI International. 